\title{
Methodology of teaching the techniques of playing volleyball among 11-12 year-old boys
}

\author{
Irina Lazunina, Maria Kosheleva* \\ Tolyatti State University, 445020, Tolyatti, Russia
}

\begin{abstract}
In all games, connected with the ball, main attention of a player should be paid to a moving ball. This rule ignoring leads to often inaccurate ball hitting. Admittedly, players should be taught to observe the opponent. But at the same time direct observation is held to the definite limit. And during the moment of direct training and hit fulfillment main attention is paid to the ball and other environment is observed only with the help of peripheral vision. During the game young volleyball players lose many favorable moments only because they can't choose the best ways of a hit in the combat with the opponent. During the playing technique explanation and study it is necessary to study thoroughly the basis of the technique, the sequence of the separate body parts movements, speed and their amplitude during different techniques fulfillment. These factors are the main for volleyball player's actions effectiveness achievement. While the attacking hit fulfillment and blocking it is important to control the opponent during the technique fulfillment. That is why it is necessary to master the ability to observe the opponent's movements all the time, till the hit on the ball, among young volleyball players.
\end{abstract}

\section{Introduction}

Using volleyball in physical upbringing of youth has the range of advantages in comparison with other sports games use: in volleyball people can play outdoors, the game doesn't demand great territories, doesn't claim great demands on equipment, different amount of players or mixed teams can play. The rules of the game are clear, they can be adopted depending on conditions [1].

During the basis of volleyball playing technique formation it is necessary to work methodically correct and sequentially. It is possible owing to the methods of analysis and synthesis, by means of sports game consideration according to the separate elements of techniques estimation, their significance and difficulty estimation. In terms of volleyball specificity great demands are claimed on a player, connected with perfect movements coordination. Educational-training process with a young volleyball player should be planned correctly, as different injuries. They affect the lower part of spine, knee and shoulder, are possible in volleyball. These injuries present the source of disability and working capacity worsening among professional volleyball players [2].

We supposed that developing the methodology of teaching the techniques of playing volleyball, we would solve the main objectives of technical training and an effective use of the received knowledge and skills during the competitions. The process provided the main problem solution: to create and introduce the methodology of playing volleyball teaching techniques in 11-12 year-old boys.

Thoroughly prepared player has advantages over the volleyball player, who pays his attention only to the technique of ball use in his training process. That is why beginners should understand the importance of mastering all their movements. During teaching the techniques players master necessary technical skills of the game and on their basis master more difficult techniques. They are necessary during the game and during the competitions.

\section{Materials and methods}

During the pedagogical work we used traditional research methods: scientific sources analysis, pedagogical observation, pedagogical experiment, control tests, methods of mathematical statistics. The pedagogical experiment was held on the basis of the municipal budgetary establishment school №2 sports gym in Tolyatti, Russia. Two groups were formed: the control group. They included boys, who train according to generally adopted program and the experimental group of boys. They trained according to the created methodology. The pedagogical experiment was held with 11-12 year-old boys (12 people in each group-control and experimental ones). All boys were from the basic medical group. During the week the boys from the control and the experimental groups trained twice a week. The duration of each lesson was 45 minutes.

The specificity of the experimental methodology:

- getting the boys acquainted with the studied techniques (the technique demonstration should be correct and should be with examples);

- it is necessary to choose correctly the supplying exercises, close to the technique in its structure;

* Corresponding author: mar980@yandex.ru 
- mastering can be held in conditions close to game situation, it can be provided with the help of repeated repetitions during the educational games. It provides their correct fulfillment in quickly changing conditions.

Considering the questions of study succession, we took into account the following:

- Firstly, the importance of the separate techniques and tactical actions form the point of view of their effectiveness in game;

- Secondly, logic connection of game techniques in attack and defense, tactical actions in attack and defense;

- Thirdly, the necessity to connect the technique and tactics in teaching, the ability to realize interconnection between technical and tactical training [3].

Pashkova N.V. mentions the following: "Motor actions optimization is one of the urgent objectives of the theory and methodology of sports training and constant level of sports achievements increase causes the necessity to search for new, more effective ways of sports training" [4]. In this connection, the exercises, directed toward the separate parts of the technique and the details of the technique, should be constantly combined with the technique fulfillment in general, with individual and group exercises.

\section{Results and Discussion}

Control over the degree of mastering the techniques is realized during observations. Novik E. mentions the following: "In the program of physical upbringing volleyball plays an important role. In this connection there appears the need for the held lessons effectiveness improvement. It is connected with the search for new ways of educational-training process organization" [5]. During the pedagogical experiment the coach writes in a special register during which lesson a young volleyball player mastered this or that phase of the technique, the base of the technique in general (it means he fulfilled it confidently out of game environment). Time, which a young volleyball player spends on mastering the technique, is one of the indices of a volleyball player's abilities. All respondents should master the whole volume of program material. The higher the level of special training is, the higher is the confidence of the players and the team in general.

Special abilities and qualities development provides quicker mastering rational technique and successful formation of tactical skills. All players in a team should act as one unit, in general desire to achieve the main aim and be in a good physical fitness. During the pedagogical experiment the boys went through control normatives, which characterized their general physical readiness.

Table 1. The results of boys' physical readiness during the pedagogical experiment

\begin{tabular}{|c|c|c|c|}
\hline Control exercises & CG & EG & P \\
\hline Shuttle run 3X10, s & $8,8 \pm 0,7$ & $8,6 \pm 0,9$ & $\leq 0,05$ \\
\hline 30 m running, s & $6,0 \pm 1,2$ & $5,8 \pm 0,9$ & $\leq 0,05$ \\
\hline 60 m running, s & $11,0 \pm 0,9$ & $10,8 \pm 0,3$ & $\leq 0,05$ \\
\hline Standing long-jump, cm & $155,0 \pm 2,1$ & $160,3 \pm 2,1$ & $\leq 0,05$ \\
\hline Triple jump, cm & $410,2 \pm 2,4$ & $420,8 \pm 2,1$ & $\leq 0,05$ \\
\hline $\begin{array}{c}\text { Standing vertical jump with } \\
\text { hands swing, cm }\end{array}$ & $18,2 \pm 0,7$ & $20,2 \pm 0,8$ & $\leq 0,05$ \\
\hline
\end{tabular}

Using and mastering the games, in the content of which techniques were practiced, had a positive influence on physical readiness of the boys form the experimental group. Speed abilities in sprint and the average indices of jumping tests were higher in the boys from the experimental group. The correct choice of special physical training means and methods can provide an optimal level of specific qualities development. They are necessary for successful mastering the skills of playing volleyball. Spirin M.P. and some other authors mention the following: "Scientific and methodical literature analysis, conversations with the coach and athletes, own research works helped to reveal that changes in the game rules influenced the content of competitive activity. The changes in its structure and content should be taken into account during volleyball players' training process organization, especially the training process of the reserve. If not, the orientation and content of training wouldn't correspond with the specificity of competitive activity and would have a negative influence on the results of competitions" [6].

A practical unit of technical training is formed by the definite skills and abilities. They help the player to act individually or together with team partners. The results of competitive activity in volleyball show that the main mistakes during the game are made by athletes during their interaction, in the average the percentage of mistakes is $60 \%$ of all possible mistakes in the game. It is connected with weak interaction between the players and game situations prediction in volleyball during the game. The main objective of female volleyball players' technical training development is the following: difficult reactions quickness, orientation, quick-wittedness development. It is mainly solved during physical and technical training. For this purpose we introduce additional conditions and tasks into educational-training process of physical training or technique. These additional conditions and tasks give the exercises tactical characteristics [7].

Correction of mistakes is a realized work of a player for own disadvantages elimination under the direction of a coach. The aim of a coach is to notice the mistake and explain the player, how to correct it. The exercises repetition helps to master correct skills. For example, if the blocking player jumps ahead all the time, he should train near the wall, gradually moving the line of a push to the wall. Jumping mastering is included into the main and preparatory parts of the lessons. Defender should be always ready for the attack. That is why it is necessary to learn to fall 
correctly in order not to have injuries. Correctly held fall should lead to a favorable initial position, out of which the player would be able to stand up quickly. In the experimental group sufficient amount of time was devoted to mistakes correction. The greatest number of mistakes is usually made by young volleyball players during hands movement, where it is important to have correct fingers position. Sometimes the beginner throws out finger-tips towards the ball: it leads to injuries; more often the beginner presses fingers to each other and in this case he incorrectly accepts the ball with the palm. The next mistake is untimely touching the ball. The player throws out hands too late or too early, then hits the ball with too bent hands or with straight hands, where the ball stops instead of being returned. During collective exercises (in team, in a group) the method of individual mistakes control is preferable. These mistakes correction is held individually, the control of a coach during the lessons is necessary. The player should train with the ball as often as possible, in order to get used to ball (repeated hits on the wall and etc.).

Having the desire to win a team faces the opponent's resistance, in order to overcome this resistance players should not only achieve advantage in techniques, but also be able to guess the plans of the opponent, use more effective tactics and be able to achieve positive result in the game. Teams get ready for serious games beforehand, studying and observing the opponent in order to make the plan and prepare necessary tactical action, including the corresponding techniques. Players also get ready psychologically, in general training is held in order to help the players make decisions individually for more favorable action fulfillment. However, game tactics starts earlier: during players selection for the team. According to the qualities of these players suitable style of playing is chosen and a log-term plan of training is set.

Bochkareva O.A. recommends the following: "In order to increase the level of unity it is necessary to form players into pairs, trios and quads according to their game role (setter-forward, help defender-setter-forward). During the training lessons they get used to each other and then owing to momentary mutual understanding they have advantage over the opponents- understand each other" [8]. Tactical correspondence of the players groups is the base of the whole play and the tactics of the collective provides victory of the whole team. Players should understand the objective. It is set for the whole team.

During the pedagogical experiment we registered the results of the normatives fulfillment in technical training of boys. The boys from the experimental group showed better results, than the boys from the control group during the control normatives in technical training fulfillment: the second pass for accuracy from zone 3 into zone 4 , the second pass for accuracy from zone 2 into zone 4 (table 2, picture 1). It is connected with the peculiarity of the experimental methodology. In it more time was given to techniques mastering. The boys from the experimental group mastered them in pairs, trios and in combinations of different difficulty.

Table 2. The results of normatives fulfillment in technical training of boys

\begin{tabular}{|c|c|c|c|}
\hline Control normatives & CG & EG & P \\
\hline $\begin{array}{c}\text { Overhead pass near the wall, facing the wall and } \\
\text { with back position (alternation), points }\end{array}$ & $2,7 \pm 0,3$ & $3,6 \pm 0,4$ & $\leq 0,05$ \\
\hline $\begin{array}{c}\text { The second pass for accuracy from zone 3 into } \\
\text { zone 4, points }\end{array}$ & $3,2 \pm 0,2$ & $4,2 \pm 0,2$ & $\leq 0,05$ \\
\hline $\begin{array}{c}\text { The second pass for accuracy from zone 2 into } \\
\text { zone 4, points }\end{array}$ & $3,4 \pm 0,4$ & $4,4 \pm 0,2$ & $\leq 0,05$ \\
\hline Pass for accuracy: the upper line, points & $2,8 \pm 0,2$ & $4,2 \pm 0,4$ & $\leq 0,05$ \\
\hline
\end{tabular}

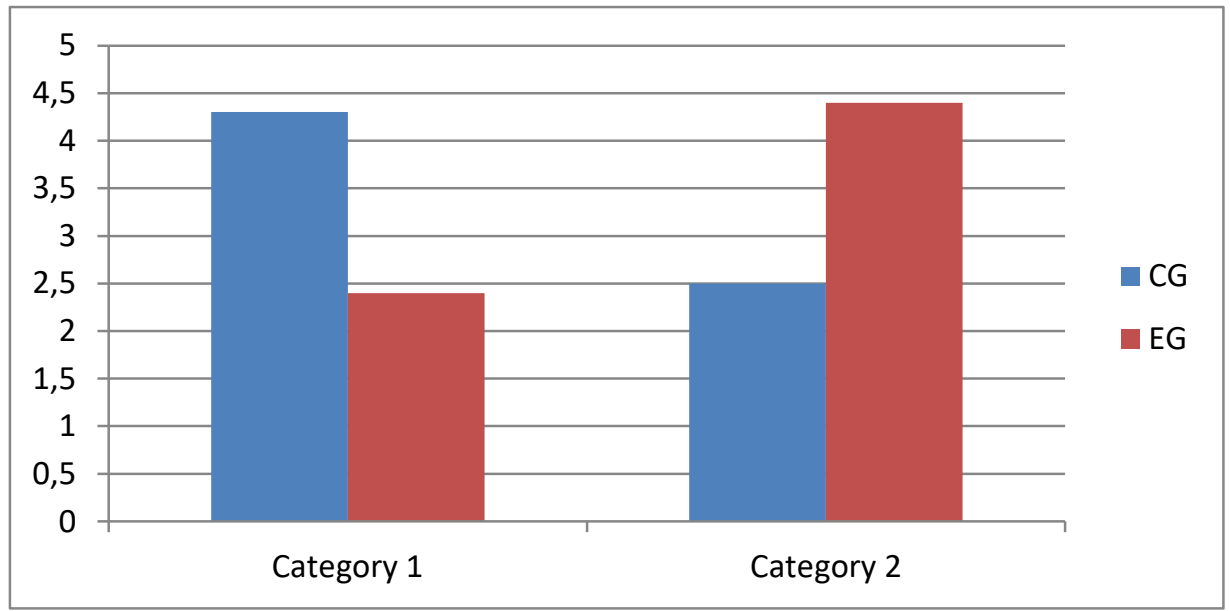

Fig. 1. Results of normatives fulfillment in technical training.

For volleyball, the game which has the character of collective competition, tactics is very important. Tactics study starts from the first steps of teaching the beginners, it is the only way for the perfect playing tactics mastering. Each beginner during the training lessons gets acquainted with the game tactics. Studying the results of control tests 
we come to the conclusion that a purposeful educational-training process for techniques mastering, using the games of different content and character, leads to the skill of playing volleyball formation, which conditions the results of the game.

Table 3. Results of normatives fulfillment in technical training of boys

\begin{tabular}{|c|c|c|c|}
\hline Control normatives & CG & EG & P \\
\hline $\begin{array}{c}\text { Pass receiving from zone 5 into zone 2 for } \\
\text { accuracy, points }\end{array}$ & $2,4 \pm 0,2$ & $3,2 \pm 0,4$ & $\leq 0,05$ \\
\hline $\begin{array}{c}\text { Pass receiving from zone 6 into zone 3 for } \\
\text { accuracy, points }\end{array}$ & $2,6 \pm 0,2$ & $3,2 \pm 0,2$ & $\leq 0,05$ \\
\hline $\begin{array}{c}\text { Single blocking of the forward from zone 4(2) } \\
\text { diagonally, points }\end{array}$ & $1,8 \pm 0,2$ & $2,2 \pm 0,2$ & $\leq 0,05$ \\
\hline
\end{tabular}

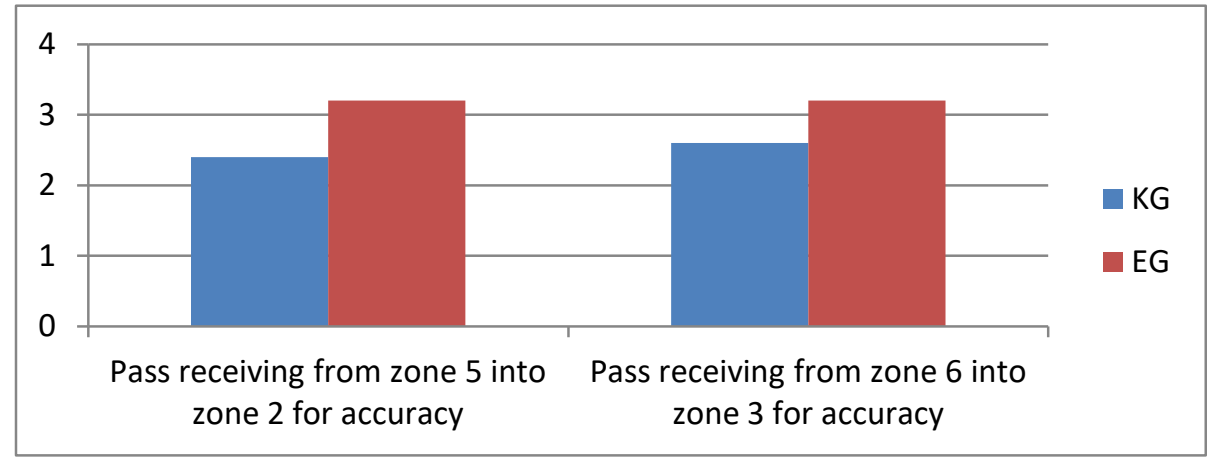

Fig. 2. Results of normatives fulfillment in technical training.

\section{Conclusions}

The integral approach to the quality of mastering techniques is perspective (complex index, in which quantitative and qualitative estimations are combined). Playing activity, on the one hand, is the starting point in means classification organization (physical exercises), used for volleyball players training, on the other hand, the final stage of playing skills formation - the skill achievement and effective use of technical-tactical arsenal in a game. While teaching we systematically alternated the forms of teaching. They have different orientation- during the elements of the technique study and in general. It is necessary to train all players in a way that if necessary they could be forwards and defenders. After that it would be easier for them to master more developed styles of modern volleyball game. Team interaction of boys are corresponded and taught in the definite tactical combinations, during the pedagogical experiment they oriented themselves well during the game in defense and attack. During educationaltraining process they developed their techniques, tactical skills and interaction with team players.

\section{References}

1. A.S. Kuznetsov, Z.M. Kuznetsova, Russian Journal of Physical Education and Sport, 14(4), 5-7 (2019). DOI: 10.14526/20704798-2019-14-4-5-7

2. R. Bahr, J.C. Reeser, American Journal of Sports Medicine, 31(1), 119 (2003)

3. I.F. Mezhman, Learn to play volleyball: manual (Volga region State University of telecommunications and informatics, Samara, 2017)

4. N.V. Pashkova, Bulletin of Tomsk State Pedagogical University, 8(86), 75-78 (2009)

5. E. Novik, Theory and practice of physical culture, 12, 42-45 (2007)

6. M.P. Spirin, G.Ya. Shipulin, O.E. Serdyukov, L.V. Zhilina, O.V. Chernykh, Theory and practice of physical culture, 9, $34-$ 37 (2007)

7. I.V. Lazunina, World of science, culture, education, 5(66), 164-165 (2017)

8. O.A. Bochkareva, Theory and practice of physical culture, 9, 27-29 (2008) 